\title{
Ivosidenib in relapsed or refractory acute myeloid leukemia: a profile of its use in the USA
}

\author{
Emma H. McCafferty ${ }^{1} \cdot$ Katherine A. Lyseng-Williamson $^{1}$ (1)
}

Published online: 18 February 2019

๑) Springer Nature 2019, corrected publication 2019

\begin{abstract}
Ivosidenib (Tibsovo ${ }^{\circledR}$ ), a first-in-class, small molecule inhibitor of mutant isocitrate dehydrogenase 1 (IDH1), is an important new therapeutic option in the USA for treating adults with relapsed or refractory acute myeloid leukemia who have a susceptible $\mathrm{IDH} I$ gene mutation. In such patients, oral ivosidenib is effective and generally well tolerated, producing encouraging rates and durations of complete remission (CR), CR or CR with partial hematologic recovery (CR/CRh), overall response, and overall survival. $\mathrm{CR} / \mathrm{CRh}$ rates are consistent across most baseline characteristics. Ivosidenib also provides additional clinical benefits, including transfusion independence, improvements in hematologic variables, and deep molecular responses as measured by $I D H 1$ mutation clearance in patients who achieve CR/CRh.
\end{abstract}

Adis evaluation of ivosidenib in the treatment

of relapsed or refractory IDHI-mutated acute

myeloid leukemia

Reduces elevated levels of D-2-hydroxyglutarate and reverses the inhibition of myeloid blast differentiation imposed by mutant IDH1 enzymes

Provides beneficial effects on rates and duration of $\mathrm{CR}$, $\mathrm{CR} / \mathrm{CRh}$, overall response, and overall survival

Improves transfusion dependence and hematologic variables

Adverse events of special interest include QT interval prolongation, IDH-differentiation syndrome, and leukocytosis; are managed using standard-of-care measures and dosage modifications, where required

Convenient once-daily oral administration regimen

Katherine A. Lyseng-Williamson

dtp@adis.com

1 Springer, Private Bag 65901, Mairangi Bay, 0754 Auckland, New Zealand

\section{What is the rationale for using ivosidenib in relapsed or refractory AML?}

Acute myeloid leukemia (AML) is a group of heterogeneous hematologic malignancies characterized by the clonal expansion of myeloid precursor cells (i.e. blasts) in the bone marrow, blood, and/or other tissues [1]. It typically arises from somatic mutations [e.g. isocitrate dehydrogenase 1 (IDHI) gene mutations] that alter the normal mechanisms of proliferation, survival, differentiation, and/or self-renewal of the blasts $[2,3]$. The pathogenesis may also involve cytogenetic alterations and/or dysregulated epigenetic mechanisms $[4,5]$. AML is diagnosed based on the presence of $\geq 20 \%$ of blasts in the bone marrow or blood; however, accurate classification requires multidisciplinary diagnostic studies [1]. AML is the most common type of acute leukemia in adults [1], with $>50 \%$ of patients being diagnosed aged $\geq 65$ years [6].

Mutations in the $I D H I$ gene are found in $\approx 6-16 \%$ of patients with AML [7]. IDHI encodes a key metabolic enzyme, IDH1, which is located in the cytoplasm and peroxisomes and catalyzes the reversible NADP+-dependent oxidative decarboxylation of isocitrate to yield $\alpha$-ketoglutarate [8]. Other metabolic IDH enzymes, namely IDH2 and IDH3 (encoded by $I D H 2$ and $I D H 3$ ) are located in the mitochondria [8]. Mutations in IDHI result in neomorphic enzymatic activity, leading to the conversion of NADH and $\alpha$-ketoglutarate to NADP+ and D-2-hydroxyglutarate (D-2HG) [8-10]. Mutant IDH1 enzymes produce high levels 
of D-2HG in cells [10]; the cellular accumulation of D-2HG competitively inhibits $\alpha$-ketoglutarate-dependent enzymes, which prevents normal hematopoietic differentiation and promotes tumorigenesis [11-13]. Patients with $I D H 1$ - or IDH2-mutated AML are associated with high rates of cooccurring NPM1 mutations, intermediate-risk cytogenetics, increased platelet counts, high numbers of bone marrow blasts at diagnosis, and older patient age [14]. The prognostic significance of $I D H 1$ or $I D H 2$ mutations in AML (i.e. compared with the wild-type $I D H$ form of the disease) remains controversial [1].

Standard treatment protocols for AML typically comprise induction chemotherapy (e.g. cytarabine and an anthracycline in patients aged $<60$ years) followed by post-remission therapy, as well as supportive care [1]. The induction therapy strategy is influenced by patient age, history of prior myelodysplasia or cytotoxic therapy, and the presence of comorbid conditions that may affect performance status [1]. In patients that are not eligible for standard induction therapy (e.g. elderly patients or those who cannot tolerate intensive chemotherapy), other therapeutic strategies may be implemented, such as single-agent chemotherapy, hypomethylating-agent based therapy (e.g. azacytidine), or best supportive care (e.g. hydroxyurea or transfusion support) [1]. Despite these methods, patient outcomes in AML are generally poor; the estimated survival rate of the disease is only $27.4 \%$ within 5 years of diagnosis [6]. The rates of achieving complete remission (CR) rarely exceed $70 \%$ in patients aged $<60$ years and $50 \%$ in patients $>60$ years [1]. Moreover, most patients who achieve CR will have a disease relapse within 3 years of diagnosis [15].

Substantial efforts have recently been made to develop novel cancer treatments that molecularly target the genomic profile of the individual, thereby improving clinical outcomes and preventing toxicities associated with standard chemotherapy $[16,17]$.

One such novel agent is oral ivosidenib (Tibsovo ${ }^{\circledR}$ ), a first-in-class, potent, selective small molecule inhibitor of susceptible mutant IDH1 enzymes developed to treat patients with cancers harboring IDHI mutations [18-20]. In vitro and in vivo, clinically relevant concentrations of ivosidenib inhibit the function of mutant, but not wild-type, IDH1 enzymes, thereby reducing elevated D-2HG levels, and reversing the inhibition of myeloid blast differentiation [18-20].

\section{When is ivosidenib therapy indicated in the USA?}

Oral ivosidenib is approved in the USA for the treatment of adults with relapsed or refractory AML who have a susceptible $I D H I$ mutation in their blood and bone marrow as detected by an FDA-approved test (e.g. the Abbott RealTime $I D H I$ test) [18]. Susceptible $I D H I$ mutations are defined as those that lead to the increase of D-2HG levels in leukemia cells, and where the efficacy of ivosidenib is predicted by clinically meaningful remissions and/or inhibition of the mutant IDH1 enzyme activity at concentrations that are sustainable at the recommended dosage, according to validated methods. The most common IDHI mutations that are susceptible to ivosidenib are $\operatorname{Arg}^{132}$ to His or Cys substitutions (i.e. R132H or R132C). Notably, patients without $I D H I$ mutations at diagnosis should be retested at relapse as $I D H I$ mutations may emerge during treatment or relapse [18].

Table 1 provides an overview of the use of ivosidenib in the treatment of AML based on the US prescribing information [18]. Dosage adjustments may be required to manage some treatment-related adverse effects (Table 1), but are not required on the basis of age, race, sex, body weight, Eastern Cooperative Oncology Group performance status (ECOGPS) score, mild or moderate renal impairment, or mild hepatic impairment (data in patients with severe renal and moderate and severe hepatic impairment are lacking) [18].

Pregnant women should be advised of the potential risk to the fetus with ivosidenib therapy (based on animal embryofetal toxicity studies); breast-feeding women should also be advised to avoid breast-feeding during ivosidenib treatment and for $\geq 1$ month after the last dose. Consult local prescribing information for further details on the use of ivosidenib [18].

\section{What are the clinically relevant effects of ivosidenib in relapsed or refractory AML?}

An open-label, dose-escalation $(n=78)+$ expansion phase ( $n=180)$, multicenter study of oral ivosidenib was conducted in a total of 258 adult patients with IDHI-mutated advanced hematological malignancies, including 179 patients with relapsed or refractory AML [21]. In the doseescalation phase, patient cohorts received ivosidenib at dosages ranging from $100 \mathrm{mg}$ twice daily to $300-1200 \mathrm{mg}$ once daily. Based on the available data regarding the safety, pharmacokinetic, and efficacy profiles of ivosidenib, the dosage that was selected to be evaluated in the expansion phase was ivosidenib $500 \mathrm{mg}$ once daily. Ivosidenib was administered in 28-day cycles [21].

\section{Pharmacodynamic effects}

D-2HG was maximally inhibited in plasma and bone marrow by day 14 in patients who received ivosidenib $500 \mathrm{mg}$ once daily, and D-2HG suppression was subsequently maintained throughout treatment [21]. Of note, mean plasma D$2 \mathrm{HG}$ decreased to the extent that was comparable to those 
Table 1 Overview of the US prescribing information of oral ivosidenib $\left(\right.$ Tibsovo $^{\circledR}$ ) in the treatment of adults with relapsed or refractory acute myeloid leukemia and a susceptible IDHI mutation in their blood or bone marrow as detected by an FDA-approved test [18]

How is ivosidenib available and how should it be administered?

Availability

Usual dosage

Film-coated tablets containing $250 \mathrm{mg}$ of ivosidenib (take whole; do not split or crush)

$500 \mathrm{mg}(2 \times 250 \mathrm{mg}$ tablets $)$ once daily at approximately the same time each day

Take with or without food; do not take with a high-fat meal ( $\uparrow$ exposure to ivosidenib by $98 \%$ )

Treatment duration

$\geq 6$ months to allow for clinical response (unless disease progression or unacceptable toxicity occurs)

What precautions should be taken with regard to the risk/occurrence of QTc interval prolongation?

Monitoring

Perform ECGs at least once weekly during the first 3 weeks of treatment, then at least once monthly

If QTc prolongation occurs and subsequently resolves, monitor ECG at least once weekly for 2 weeks

Monitor and supplement electrolytes levels as clinically indicated

$\uparrow$ QTc interval

$>480$ to $500 \mathrm{~m}$ with no signs/symptoms of life-threatening arrhythmia: interrupt ivosidenib; resume at $500 \mathrm{mg} /$ day when QTc interval returns to $\leq 480 \mathrm{~ms}$

$>500 \mathrm{~ms}$ with no signs/symptoms of life-threatening arrhythmia: interrupt ivosidenib; restart at $250 \mathrm{mg} / \mathrm{day}$ when QTc interval returns to $\leq 480 \mathrm{~ms}$ or within $30 \mathrm{~ms}$ of baseline; consider $\uparrow$ to $500 \mathrm{mg} /$ day if an alternative cause of $\uparrow$ QTc interval is identified

Signs/symptoms of life-threatening arrhythmia: permanently discontinue ivosidenib

Concomitant use of drugs that Avoid co-administration of antiarrhythmics, fluoroquinolones, 5 - $\mathrm{HT}_{3}$ receptor antagonists, triazole antifungals, may $\uparrow$ QTc interval etc. (additional risk of $\uparrow$ QTc interval)

Strong/moderate CYP3A4 inhibitors

Consider alternative therapies ( $\uparrow$ in exposure to ivosidenib leading to additional risk of $\uparrow$ QTc interval)

If concomitant use of strong CYP3A4 inhibitors is unavoidable: $\downarrow$ ivosidenib to $250 \mathrm{mg} / \mathrm{day}$

What precautions should be taken with regard to the risk/occurrence of other specific adverse effects?

Monitoring

Monitor creatine phosphokinase levels once weekly during month 1

Assess blood counts/chemistries before and during treatment (at least once weekly during month 1 , every 2 weeks during month 2 , then once monthly)

IDH differentiation syndrome Initiate systemic corticosteroids (e.g. intravenous dexamethasone $10 \mathrm{mg}$ every $12 \mathrm{~h}$ ) for $\geq 3$ days and hemodynamic monitoring until improvement; if severe signs/symptoms persist $\geq 48 \mathrm{~h}$, interrupt ivosidenib and resume when signs/symptoms improve to $\leq$ grade 2

Non-infectious leukocytosis Initiate hydroxyurea ( \pm leukapheresis): if no improvement, interrupt ivosidenib treatment; resume when leukocytosis has resolved

Guillain-Barré syndrome

Assess patients for new signs and symptoms of motor and/or sensory neuropathy

If Guillain-Barré syndrome occurs, permanently discontinue ivosidenib

Other grade 3/4 drug-related toxicity

Interrupt ivosidenib treatment; resume at a $\downarrow$ dosage of $250 \mathrm{mg} /$ day when toxicity resolves to $\leq$ grade 2 ; may $\uparrow$ to $500 \mathrm{mg} /$ day if toxicities resolve to $\leq$ grade 1

If grade $3 / 4$ toxicity recurs: permanently discontinue ivosidenib

\section{What is the pharmacokinetic profile of ivosidenib?}

Time to $\mathrm{C}_{\max }$

$\approx 3 \mathrm{~h}$ (median)

Metabolism

Primarily by CYP3A4 with minor contributions by hydrolytic and N-dealkylation pathways

Excretion

77 and $17 \%$ in feces and urine after a single dose (67 and 10\% as unchanged drug)

Terminal half-life

$93 \mathrm{~h}$

What clinically relevant pharmacokinetic drug interactions may potentially occur with ivosidenib?

Strong CYP3A4 inducers

Sensitive CYP3A4/CYP2C9

substrates
Avoid co-administration ( $\downarrow$ in exposure to ivosidenib)

Use alternative therapies ( $\downarrow$ in exposure to substrates)

If co-administration is unavoidable, monitor patients for $\downarrow$ efficacy

Do not use with CYP3A4 substrate azole antifungals (their efficacy is expected to $\downarrow$ )

Hormonal contraceptives: consider use of alternative contraceptives (efficacy of contraceptive may $\downarrow$ )

$C_{\text {max }}$ peak plasma concentration, $C Y P$ cytochrome P450, IDH isocitrate dehydrogenase, $Q T c$ corrected QT, $\uparrow$ increase(d), $\downarrow$ decrease(d)

reported in healthy individuals. No additional D-2HG inhibition was observed at higher dosages of 800 or $1200 \mathrm{mg}$ once daily. Although the suppression of D-2HG formation is indicative of pharmacologic inhibition of mutated IDH1, the degree of suppression and baseline levels of D-2HG do not predict the clinical response to ivosidenib [21]. 


\section{Clinical efficacy}

In the phase 1 trial [21], the clinical efficacy of oral ivosidenib $500 \mathrm{mg}$ once daily was assessed in 125 patients with relapsed or refractory IDH1-mutated AML. To be eligible to be included in this primary efficacy population, patients were required to have [21]:

- IDH1 mutation-positive AML that had relapsed (i.e. second or later relapse, a relapse following a stem-cell transplant, or a relapse $\leq 1$ year after initial therapy) or was refractory (i.e. refractory to induction or re-induction chemotherapy);

- received a starting dosage of ivosidenib $500 \mathrm{mg}$ once daily, with the first dose being administered $\geq 6$ months prior to the analysis cut-off date (12 May 2017);

- a baseline ECOG-PS score of 0-2;

- documented IDHI mutation(s) based on prospective central testing (which were retrospectively confirmed using an FDA-approved test for ivosidenib treatment selection [18]).

In the primary efficacy population, the median patient age was 67 years (range 18-87), 52\% were male, and 66\% had primary AML. Patients had received a median of 2 previous therapies (range 1-6), had an intermediate (53\%) or poor $(30 \%)$ cytogenetic risk status (status unknown for the remaining patients), and had received ivosidenib for a median of 3.9 months (range 0.1-25.8 months) [21].

The clinical efficacy of ivosidenib was assessed using the modified 2003 International Working Group response criteria for AML [22], where the primary endpoint was the rate of CR (defined as $<5 \%$ bone marrow blasts combined with an absolute neutrophil count of $>1 \times 10^{9} / \mathrm{L}$ and platelet count of $>100 \times 10^{9} / \mathrm{L}$ ) as assessed by the investigators, or $\mathrm{CR}$ with partial hematologic recovery $(\mathrm{CRh}$; defined as $<5 \%$ bone marrow blasts combined with an absolute neutrophil count of $>0.5 \times 10^{9} / \mathrm{L}$ and a platelet count of $>50 \times 10^{9} / \mathrm{L}$ ) as assessed by the sponsor [21] (hereafter referred to as a $\mathrm{CR} / \mathrm{CRh}$ response). Other clinical endpoints were also assessed [21]. Unless otherwise stated, results discussed are in the primary efficacy population.

\section{Clinical response}

Ivosidenib $500 \mathrm{mg}$ once daily provided encouraging rates and durations of $\mathrm{CR} / \mathrm{CRh}, \mathrm{CR}$, and overall response (Table 2) [21]. Notably, out of the patients who achieved a best response of $\mathrm{CR} / \mathrm{CRh}$, all achieved their first CR/CRh response within 6 months of initiating ivosidenib therapy [18]. Subgroup analyses indicated that CR/CRh response rates were consistent across all baseline demographics and disease characteristics, except for the number of prior regimens: patients who had received one prior regimen had a higher $\mathrm{CR} / \mathrm{CRh}$ response rate $(46 \%)$ than those who had received $\geq 3$ prior regimens (15\%) [21].

$\mathrm{CR}$ and $\mathrm{CRh}$ response rates and durations in the 125 patients in the primary efficacy population were similar to those observed in the total population of 179 patients with relapsed or refractory AML (i.e. primary efficacy population plus those who did meet the above relapsed/refractory AML eligibility criteria) [21]. Moreover, in the 101 patients with $\geq 1$ baseline co-mutations (e.g. DNMT3A, NPM1, and/ or $S R S F 2$ ) receiving ivosidenib $500 \mathrm{mg}$ once daily in the expansion phase, the mean number of co-occurring mutations was significantly lower in patients with a $\mathrm{CR} / \mathrm{CRh}$ response than in those without such a response (1.8 vs 2.6 co-mutations; $p=0.0017)$. Although mutations in receptor tyrosine kinase pathway genes (e.g. NRAS, KRAS, LT3, KIT, NF1, PTPN11, BRAF, MAP2K1, and RET) occurred significantly $(p=0.003)$ more frequently in patients who did not achieve $\mathrm{CR}$ or $\mathrm{CRh}$ than those who did achieve $\mathrm{CR}$ or $\mathrm{CRh}$ as a best response, no specific preexisting single gene mutation was deemed a significant predictor of clinical response or resistance following treatment with ivosidenib [21].

\section{Overall survival}

Treatment with once-daily ivosidenib $500 \mathrm{mg}$ was associated with encouraging rates and duration of overall survival [21]. Median overall survival was 8.8 months with a median follow-up time of 14.8 months (range 0.2-30.3 months), and in patients who achieved a $\mathrm{CR} / \mathrm{CRh}$ response, the survival rate was $50.1 \%$ after 18 months of ivosidenib therapy (median not reached by the cut-off date).

Estimates of the median overall survival were 9.3 months in patients who achieved non-CR/CRh responses (i.e. CR with incomplete hematologic or platelet recovery,

\begin{tabular}{|c|c|c|c|}
\hline \multirow[t]{2}{*}{ Outcome } & \multirow{2}{*}{$\begin{array}{l}\% \text { of patients } \\
(n=125)\end{array}$} & \multicolumn{2}{|c|}{ Median no. of months (range) } \\
\hline & & Time to response & $\begin{array}{l}\text { Response } \\
\text { duration }\end{array}$ \\
\hline $\mathrm{CR} / \mathrm{CRh}$ & $30.4^{\mathrm{a}}$ & $2.7(0.9-5.6)$ & 8.2 \\
\hline $\mathrm{CR}$ & 21.6 & $2.8(0.9-8.3)$ & 9.3 \\
\hline $\mathrm{OR}^{\mathrm{b}}$ & 41.6 & $1.9^{c}(0.8-4.7)$ & 6.5 \\
\hline
\end{tabular}

$C R$ complete remission, $C R h$ complete remission with partial hematologic recovery, $O R$ overall response

${ }^{\text {a }}$ Primary efficacy outcome

b Included CR, CR with incomplete hematologic or platelet recovery, partial remission, or morphologic leukemia-free state

${ }^{\mathrm{c}}$ Time from first dose to the first occurrence of any response (in patients who achieved a response) 
a morphologic leukemia-free state that did not meet the criteria for CRh, and/or partial remission), and 3.9 months in patients who did not achieve a response (i.e. stable or progressive disease) [21].

\section{Transfusion independence}

In addition to the rate and duration of CR/CRh response, US approval of ivosidenib $500 \mathrm{mg}$ once daily was also based on the rate of conversion from transfusion dependence to transfusion independence [18]. Following treatment with ivosidenib, the acquisition and maintenance of transfusion independence was observed across all response categories [21]. Of the 84 patients dependent on platelet and/or red blood cell transfusion at baseline, $35 \%$ became transfusion independent for $\geq 56$ days during ivosidenib therapy. Of the 41 patients not dependent on transfusions at baseline, $56 \%$ remained transfusion independent for $\geq 56$ days during ivosidenib therapy [21].

Ivosidenib $500 \mathrm{mg}$ once daily reduced the mean percentage of bone marrow blasts over time $(\approx 50 \%$ at baseline vs $\approx 10 \%$ after twelve 28 -day cycles of ivosidenib), and induced myeloid differentiation and trilineage hematopoietic recovery without a period of bone marrow aplasia (consistent with the drug's mechanism of action) [21]. Improvements from baseline in hematologic variables were also observed over time, where the mean platelet count increased from $\approx 60$ to $130 \times 10^{9} / \mathrm{L}$ and the mean absolute neutrophil count increased from $\approx 0.5$ to $\approx 3.3 \times 10^{9} / \mathrm{L}$ after $\leq 12$ cycles of ivosidenib therapy [21]. Patients who achieved a CR/CRh response had lower rates of exposure-adjusted febrile neutropenia (any grade) and grade 3/4 infections during ivosidenib therapy than with other responses [21].

\section{Mutation clearance}

IDHI mutation clearance was defined as the inability to detect a mutated IDHI ( $\mathrm{mIDHI}$ ) variant allele [variant allele frequency (VAF) cutoff of $1 \%$ as assessed using the next-generation sequencing (NGS) FoundationOne ${ }^{\mathrm{TM}}$ Heme Panel] in bone marrow mononuclear cells (BMMCs) at $\geq 1$ time point during the study, using highly sensitive digital polymerase-chain-reaction technology [21]. Of the 31 patients in the expansion phase who achieved a best response of $\mathrm{CR}$ or $\mathrm{CRh}$ and who had available longitudinal $\mathrm{mIDHI}$ VAF data for BMMCs and neutrophils, 23\% (7 patients) had clearance of $I D H I$ mutations in BMMCs following treatment with ivosidenib $500 \mathrm{mg}$ once daily. Over time, mean levels of IDH1 mutations in bone marrow mononuclear cells and neutrophils decreased in these patients. Clearance of IDH1 mutations was not observed in any patient who did not achieve $\mathrm{CR} / \mathrm{CRh}$ with ivosidenib $500 \mathrm{mg}$ once daily $(n=33)$ [21].

Moreover, preliminary findings suggest that patients with clearance of $I D H I$ mutations in BMMCs may have longer durations of response and overall survival than those without clearance [21]. The median duration of CR/CRh and overall survival were 11.1 and 14.5 months in patients with $I D H I$ mutation clearance, whereas, in patients who did not have IDHI mutation clearance, the corresponding values were 6.5 and 10.2 months [21].

\section{What is the tolerability profile of ivosidenib?}

Oral ivosidenib $500 \mathrm{mg}$ once daily had a generally manageable tolerability profile in the treatment of IDHI mutationpositive relapsed or refractory AML in the phase 1 trial [21]. Out of the 179 patients who received a starting dosage of $500 \mathrm{mg}$ once daily (safety population), $98.9 \%$ experienced treatment-emergent adverse events. The most common treatment-emergent adverse events were diarrhea, leukocytosis, febrile neutropenia, nausea, and fatigue (Fig. 1a).

\section{Treatment-related adverse events}

Treatment interruption, dosage modification, and/or permanent discontinuation may be required to manage some serious/severe adverse events related to treatment with ivosidenib (Table 1). Adverse events that have been associated with ivosidenib treatment include IDH differentiation syndrome (a boxed warning in the US prescribing information [18]), corrected QT (QTc) interval prolongation, noninfectious leukocytosis, Guillain-Barré syndrome, anemia, thrombocytopenia, diarrhea, decreased platelet counts, and hypoxia.

In the phase 1 trial, $20.7 \%$ of patients in the safety population experienced grade $3 / 4$ events related to treatment with ivosidenib $500 \mathrm{mg}$ once daily (Fig. 1b). All events were manageable with appropriate interventions [21]. Temporary treatment interruption was required by $35.2 \%$ of patients, with a median duration of interruption of 8 days (range 1-36 days). Dosage reductions to manage adverse events were uncommon, being required by only six (3.4\%) patients [21]; there were two discontinuations for QTc prolongation (one serious), and one each for anemia, decreased platelet count, increased ALT, increased AST, neutropenia, thrombocytopenia, serious diarrhea, and serious nausea [21]. In the safety population, no patients permanently discontinued ivosidenib due to a treatment-related adverse event, and there were no treatment-related deaths. 
Adverse events of special interest in the safety population of the phase 1 trial were as follows [21]:

- QTc interval prolongation Treatment-emergent (any grade) reported in $24.6 \%$ of patients (Fig. 1a), and treatment-related grade $3 / 4$ in $7.8 \%$ (Fig. 1b); $7.3 \%$ of patients required treatment interruption, and $1.1 \%$ required dosage reduction. Drugs that are known to increase the QTc interval (Table 1) were often used concomitantly with ivosidenib; $59.2 \%$ of patients received levofloxacin, $39.7 \%$ ondansetron, $35.8 \%$ voriconazole, $26.3 \%$ fluconazole, $24.6 \%$ ciprofloxacin, and $21.2 \%$ posaconazole.

- Leukocytosis Treatment-emergent (any grade) reported in $29.6 \%$ of patients (Fig. 1a), and treatment-related grade $3 / 4$ in $1.7 \%$ (Fig. 1b); $2.8 \%$ of patients required treatment interruption; none required dosage reduction. The time to the first onset was generally $\leq 30$ days.

- IDH differentiation syndrome Thought to be due to the rapid increase in differentiation of neutrophils following the removal of the differentiation block in the malignant myeloid clone [23], and may be life-threatening or fatal if not treated [18]. Treatment-emergent (any grade) differentiation syndrome was reported in $10.6 \%$ of patients, and treatment-related grade 3 in $3.9 \%$ (Fig. 1b), with no grade 4 events [21]. The median time to onset was 29 days (range 5-59 days). In 19 patients who required interventions (e.g. corticosteroids, diuretics, and hydroxyurea if concurrent leukocytosis), the syndrome had resolved in $17(89.5 \%)$ by the cut-off date. Importantly, the approved US prescribing information cites a higher differentiation syndrome rate of $19 \%$ [18], which is derived from an algorithmic analysis of patient-level data from the clinical trial [24]. This analysis grouped adverse events and laboratory events using the Montesinos criteria [25].

\section{What is the current clinical position of ivosidenib in relapsed or refractory AML?}

Ivosidenib, a first-in-class small molecule inhibitor of mutant IDH1 with a convenient once-daily oral dosing regimen, is an important new option to treat adults with relapsed or refractory AML and a susceptible $I D H 1$ mutation. At the recommended dosage of $500 \mathrm{mg}$ once daily, ivosidenib produces encouraging rates and durations of $\mathrm{CR} / \mathrm{CRh}, \mathrm{CR}$, overall response, and overall survival in adult patients with $I D H 1$ mutation-positive relapsed or refractory AML. Additional clinical benefits are also observed in ivosidenib recipients in this patient population, including transfusion independence, improvements in hematologic variables, and deep molecular responses as measured by $I D H 1$ mutation clearance in patients who achieved CR/CRh.

Ivosidenib $500 \mathrm{mg}$ once daily has a generally manageable tolerability profile in adults with $I D H 1$ mutation-positive relapsed or refractory AML, with the most common adverse (a)

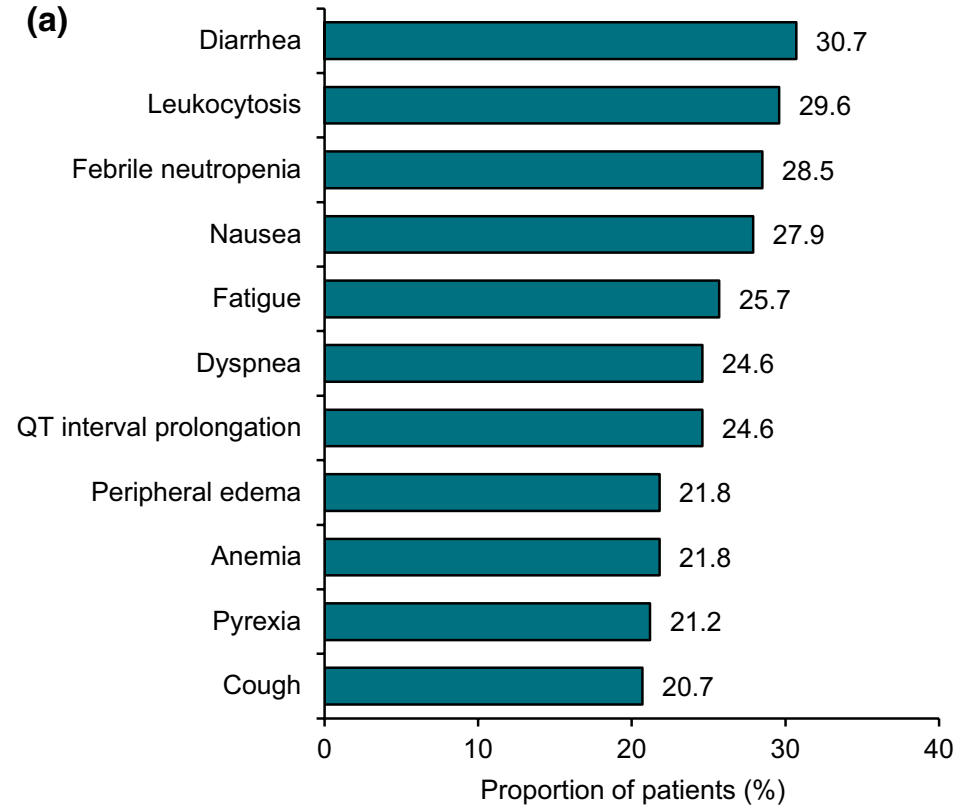

(b)

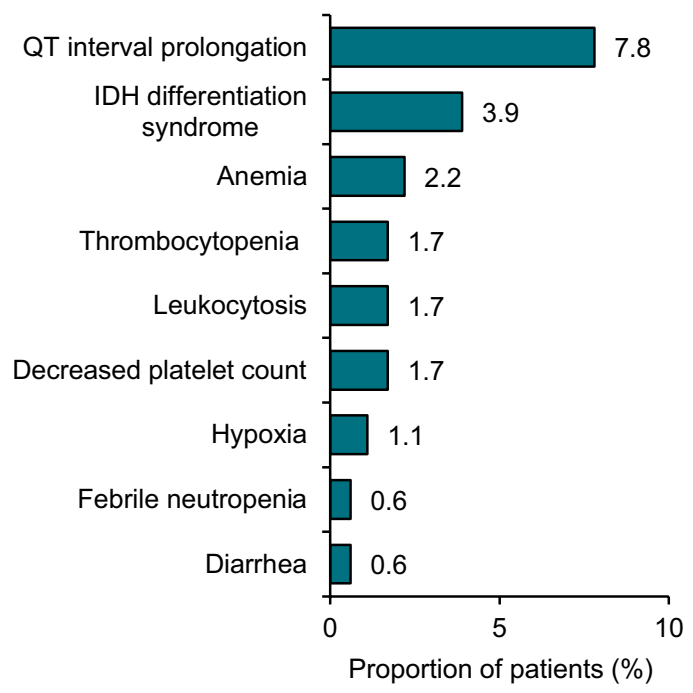

Fig. 1 Tolerability of ivosidenib in the treatment of $I D H 1$ mutationpositive relapsed or refractory acute myeloid leukemia in the safety population of 179 patients receiving a starting dosage of $500 \mathrm{mg}$ once daily in the phase 1 trial [21]: a treatment-emergent adverse events reported in $\geq 20 \%$ of patients; $\mathbf{b}$ grade $3 / 4$ treatment-related adverse events as assessed by the investigator. $I D H$ isocitrate dehydrogenase 
events being diarrhea, leukocytosis, febrile neutropenia, nausea, and fatigue, and the most common grade 3/4 treatment-related events being QT interval prolongation, IDH differentiation syndrome, and anemia. Precautions should be followed to minimize the risk of potentially serious treatment-related adverse events, and to optimize the clinical response. Treatment interruption and, less frequently, dosage modification and/or treatment discontinuation may be required to manage serious/severe adverse effects.

Acknowledgements The manuscript was reviewed by: G. N. Mannis, Hematology/Blood and Marrow Transplant, Department of Medicine, University of California, San Francisco, CA, USA; A. S. Mims, Division of Hematology, Department of Medicine, Ohio State University, Columbus, OH, USA. During the peer review process, Agios Inc., the marketing-authorization holder of ivosidenib (Tibsovo ${ }^{\circledR}$ ), was also offered an opportunity to provide a scientific accuracy review of their data. Changes resulting from comments received were made on the basis of scientific and editorial merit.

\section{Compliance with ethical standards}

Funding The preparation of this review was not supported by any external funding.

Conflicts of interest E.H. McCafferty and K.A. Lyseng-Williamson are employees of Adis/Springer, are responsible for the article content and declare no conflicts of interest.

Open Access This article is distributed under the terms of the Creative Commons Attribution-NonCommercial 4.0 International License (http://creativecommons.org/licenses/by-nc/4.0/), which permits any noncommercial use, duplication, adaptation, distribution and reproduction in any medium or format, as long as you give appropriate credit to the original author(s) and the source, provide a link to the Creative Commons license and indicate if changes were made.

\section{References}

1. National Comprehensive Cancer Network ${ }^{\circledR}$. NCCN clinical practice guidelines in oncology (NCCN guidelines ${ }^{\circledR}$ ): acute myeloid leukemia (version 3.2018). Plymouth Meeting, PA: National Comprehensive Cancer Network ${ }^{\circledR}$, Inc.; 2018.

2. Frohling S, Scholl C, Gilliland DG, et al. Genetics of myeloid malignancies: pathogenetic and clinical implications. J Clin Oncol. 2005;23(26):6285-95.

3. Marcucci G, Haferlach T, Dohner H. Molecular genetics of adult acute myeloid leukemia: prognostic and therapeutic implications. J Clin Oncol. 2011;29(5):475-86.

4. Wouters BJ, Delwel R. Epigenetics and approaches to targeted epigenetic therapy in acute myeloid leukemia. Blood. 2016;127(1):42-52.

5. Schoch C, Haferlach T. Cytogenetics in acute myeloid leukemia. Curr Oncol Rep. 2002;4(5):390-7.

6. National Cancer Institute: Surveillance, epidemiology, and End Results Program (SEER). Cancer stat facts: leukemia-acute myeloid leukemia (AML) https://seer.cancer.gov/statfacts/html/ amyl.html. Accessed Feb 12019.

7. Im AP, Sehgal AR, Carroll MP, et al. DNMT3A and IDH mutations in acute myeloid leukemia and other myeloid malignancies: associations with prognosis and potential treatment strategies. Leukemia. 2014;28(9):1774-83.

8. Mondesir J, Willekens $\mathrm{C}$, Touat $\mathrm{M}$, et al. $I D H 1$ and $I D H 2$ mutations as novel therapeutic targets: current perspectives. J Blood Med. 2016;7:171-80.

9. Ward PS, Patel J, Wise DR, et al. The common feature of leukemia-associated $I D H 1$ and $I D H 2$ mutations is a neomorphic enzyme activity converting $\alpha$-ketoglutarate to 2-hydroxyglutarate. Cancer Cell. 2010;17(3):225-34.

10. Dang L, White DW, Gross S, et al. Cancer-associated IDH1 mutations produce 2-hydroxyglutarate. Nature. 2009;462(7274):739-44.

11. Lu C, Ward PS, Kapoor GS, et al. IDH mutation impairs histone demethylation and results in a block to cell differentiation. Nature. 2012;483(7390):474-8.

12. Krell D, Mulholland P, Frampton AE, et al. IDH mutations in tumorigenesis and their potential role as novel therapeutic targets. Future Oncol. 2013;9(12):1923-35.

13. Figueroa ME, Abdel-Wahab O, Lu C, et al. Leukemic $I D H 1$ and IDH2 mutations result in a hypermethylation phenotype, disrupt TET2 function, and impair hematopoietic differentiation. Cancer Cell. 2010;18(6):553-67.

14. DiNardo CD, Ravandi F, Agresta S, et al. Characteristics, clinical outcome, and prognostic significance of $I D H$ mutations in AML. Am J Hematol. 2015;90(8):732-6.

15. Dohner H, Estey EH, Amadori S, et al. Diagnosis and management of acute myeloid leukemia in adults: recommendations from an international expert panel, on behalf of the European LeukemiaNet. Blood. 2010;115(3):453-74.

16. Bullinger L, Dohner K, Dohner H. Genomics of acute myeloid leukemia diagnosis and pathways. J Clin Oncol. 2017;35(9):934-46.

17. Schwaederle M, Zhao M, Lee JJ, et al. Impact of precision medicine in diverse cancers: a meta-analysis of phase II clinical trials. J Clin Oncol. 2015;33(32):3817-25.

18. Tibsovo ${ }^{\circledR}$ (ivosidenib) tablets, for oral use: US prescribing information. Cambridge: Agios Pharmaceuticals Inc.; 2018.

19. Popovici-Muller J, Lemieux RM, Artin E, et al. Discovery of AG-120 (ivosidenib): a first-in-class mutant IDH1 inhibitor for the treatment of IDHI mutant cancers. ACS Med Chem Lett. 2018;9(4):300-5.

20. IDH program. Cambridge: Agios Pharmaceuticals Inc.; 2018. https://agios.com/pipeline/idh-program. Accessed 1 Feb 2019.

21. DiNardo CD, Stein EM, de Botton S, et al. Durable remissions with ivosidenib in IDH1-mutated relapsed or refractory AML. N Engl J Med. 2018;378(25):2386-98.

22. Cheson BD, Bennett JM, Kopecky KJ, et al. Revised recommendations of the International Working Group for diagnosis, standardization of response criteria, treatment outcomes, and reporting standards for therapeutic trials in acute myeloid leukemia. J Clin Oncol. 2003;21(24):4642-9.

23. Birendra KC, DiNardo CD. Evidence for clinical differentiation and differentiation syndrome in patients with acute myeloid leukemia and IDH1 mutations treated with the targeted mutant IDH1 inhibitor, AG-120. Clin Lymphoma Myeloma Leuk. 2016;16(8):460-5.

24. Norsworthy JK, Mulkey F, Ward AF, et al. Incidence of differentiation syndrome with ivosidenib (IVO) and enasidenib (ENA) for treatment of patients with relapsed or refractory $(\mathrm{R} / \mathrm{R})$ isocitrate dehydrogenase (IDH)1- or IDH2-mutated acute myeloid leukemia (AML): a systematic analysis by the U.S. Food and Drug Administration (FDA) [abstract no. 288]. In: 60th American Society of Hematology Annual Meeting. 2018.

25. Montesinos P, Bergua JM, Vellenga E, et al. Differentiation syndrome in patients with acute promyelocytic leukemia treated with all-trans retinoic acid and anthracycline chemotherapy: characteristics, outcome, and prognostic factors. Blood. 2009;113(4):775-83. 